\title{
Accuracy of the Compressed Sensing Accelerated 3D-FLAIR Sequence for the Detection of MS Plaques at 3T
}

\author{
(D) S. Toledano-Massiah, (D) A. Sayadi, (D) R. de Boer, (D). Gelderblom, (D) R. Mahdjoub, (D). Gerber, (D). Zuber, (D) M. Zins,
} and (D). Hodel

\begin{abstract}
BACKGROUND AND PURPOSE: The use of 3D FLAIR improves the detection of brain lesions in MS patients, but requires long acquisition times. Compressed sensing reduces acquisition time by using the sparsity of MR images to randomly undersample the k-space. Our aim was to compare the image quality and diagnostic performance of 3D-FLAIR with and without compressed sensing for the detection of multiple sclerosis lesions at 3T.
\end{abstract}

MATERIALS AND METHODS: Twenty-three patients with relapsing-remitting MS underwent both conventional 3D-FLAIR and compressed sensing 3D-FLAIR on a 3T scanner (reduction in scan time 1 minute 25 seconds, $27 \%$; compressed sensing factor of 1.3). Two blinded readers independently evaluated both conventional and compressed sensing FLAIR for image quality (SNR and contrast-to-noise ratio) and the number of MS lesions visible in the periventricular, intra-juxtacortical, infratentorial, and optic nerve regions. The volume of white matter lesions was measured with automatic postprocessing segmentation software for each FLAIR sequence.

RESULTS: Image quality and the number of MS lesions detected by the readers were similar between the 2 FLAIR acquisitions $(P=.74$ and $P=$ .094, respectively). Almost perfect agreement was found between both FLAIR acquisitions for total MS lesion count (Lin concordance correlation coefficient $=0.99$ ). Agreement between conventional and compressed sensing FLAIR was almost perfect for periventricular and infratentorial lesions and substantial for intrajuxtacortical and optic nerve lesions. Postprocessing with the segmentation software did not reveal a significant difference between conventional and compressed sensing FLAIR in total MS lesion volume $(P=.63)$ or the number of MS lesions $(P=.15)$.

CONCLUSIONS: With a compressed sensing factor of 1.3, 3D-FLAIR is $27 \%$ faster and preserves diagnostic performance for the detection of MS plaques at 3T.

ABBREVIATIONS: CNR = contrast-to-noise ratio; $\mathrm{CS}=$ compressed sensing; MAGNIMS = Magnetic Resonance Imaging in Multiple Sclerosis; $\mathrm{PI}=$ parallel imaging

$T^{1}$ he diagnosis of MS relies on the demonstration of the dissemination of white matter hyperintensities in space and time with MR imaging. ${ }^{1}$ The FLAIR sequence plays a pivotal role in patients with MS because it shows white matter lesions in specific locations (subtentorial, optic nerve, ${ }^{2}$ juxtacortical, periventricular). ${ }^{1,3}$ In its $3 \mathrm{D}$ implementation, FLAIR also improves the detection of small demyelinating lesions, resulting in better clinicalradiologic correlation. ${ }^{4}$

Despite the proved diagnostic advantage of 3D-FLAIR versus

Received September 3, 2017; accepted after revision November 3.

From the Departments of Radiology (S.T.-M., A.S., S.G., M.Zins) and Neurology (M.Zuber), Fondation Hôpital Saint-Joseph, Paris, France; Quantib B.V. (R.d.B., J.G.), Rotterdam, the Netherlands; GE Healthcare (R.M.), Buc, France; and Department of Neuroradiology (..H.), AP-HP, Hôpitaux Universitaires Henri Mondor, Université Paris est, Créteil, France.

Please address correspondence to Sarah Toledano-Massiah, MD, Department of Radiology, Fondation Hôpital Saint-Joseph, 185 rue Raymond Losserand, 75014 Paris, France; e-mail: stoledano@hpsj.fr

http://dx.doi.org/10.3174/ajnr.A5517
2D-FLAIR, the longer scan time of the 3D version has so far hindered its adoption.

Compressed sensing (CS) is an acceleration technique newly available in MR imaging clinical routine. It uses the sparsity of MR images to randomly undersample the $k$-space, thus saving scan time. ${ }^{5-9}$ Contrary to parallel imaging (PI), CS is insensitive to the coil configuration. Because each acceleration technique imposes independent constraints on the image reconstruction, CS and PI can be used concurrently. ${ }^{10}$ The combination of PI and CS we use is serial. This feature makes the 2 acceleration techniques independent of one another; therefore, the PI sensitivity to coil configuration does not impact CS. ${ }^{11}$

There is no study yet regarding the effect of CS on diagnostic performance when applied to accelerate 3D-FLAIR acquisitions in patients with MS, to our knowledge. The aim of this study was to compare both image quality and diagnostic performance of 3D-FLAIR with and without CS in the clinical setting of MS. 
Table 1: Imaging parameters for the CS and conventional 3D-FLAIR

\begin{tabular}{|c|c|c|}
\hline \multicolumn{3}{|l|}{ Parameters } \\
\hline \multicolumn{3}{|l|}{ CS 3D-FLAIR } \\
\hline $\mathrm{TE} / \mathrm{TI} / \mathrm{TR}$ (ms) & \multicolumn{2}{|c|}{$140 / 2064 / 8000$} \\
\hline ETL & \multicolumn{2}{|l|}{220} \\
\hline FOV (frequency $\times$ phase) $(\mathrm{mm})$ & \multicolumn{2}{|c|}{$260 \times 221$} \\
\hline Slice thickness (mm) & \multicolumn{2}{|c|}{$1.2 \mathrm{~mm}$} \\
\hline Matrix (frequency $\times$ phase) & \multicolumn{2}{|c|}{$288 \times 256$} \\
\hline NEX & \multicolumn{2}{|c|}{1} \\
\hline Bandwidth ( $\mathrm{Hz} /$ pixel) & \multicolumn{2}{|c|}{347.2} \\
\hline ARC factor (phase $\times$ slice) & \multicolumn{2}{|c|}{$2.0 \times 2.0$} \\
\hline \multicolumn{3}{|l|}{ CS 3D-FLAIR/conventional 3D-FLAIR } \\
\hline CS factor & 1.3 & NA \\
\hline Scan time (min:sec) & $3: 50(-27 \%)$ & 5:15 \\
\hline
\end{tabular}

Note:-ETL indicates echo-train length; ARC, auto-calibrating reconstruction for Cartesian imaging (acceleration using parallel imaging technique); NA, not applicable. ${ }^{a} \mathrm{CS}$ allowed a $27 \%$ reduction in scan time of the 3D-FLAIR sequence.

\section{MATERIALS AND METHODS \\ Patients and MR Imaging}

This study was approved by our institutional review board, at Fondation Hôpital Saint Joseph. Informed consent was obtained from all patients. From January 2017 to April 2017, twenty-three consecutive patients with relapsing-remitting MS according to the revised McDonald criteria ${ }^{1}$ ( 14 women; mean age, 47 years; range, 21-77 years) underwent MR imaging for their routine follow-up (3T Discovery MR750; GE Healthcare, Milwaukee, Wisconsin) using a 32-channel head coil.

MR imaging protocol included DWI, 3D gradient-echo magnetization-prepared T1-weighted BRAVO (GE Healthcare; isotropic voxel, $0.9 \mathrm{~mm}$ ), T2WI, and 3D-FLAIR imaging, without administration of contrast agent. The 3D-FLAIR sequence was performed twice with and without the addition of CS. A CS acceleration factor of 1.3 was used, leading to a $27 \%$ reduction in scan time ( 1 minute 25 seconds). Both FLAIR sequences were strictly identical except for the addition of CS. All 3D-FLAIR, with and without CS, were acquired in the sagittal plane with a PI acceleration auto-calibrating reconstruction for Cartesian imaging factor of 2.

Imaging parameters are summarized in Table 1.

\section{Preliminary Study in Healthy Volunteers}

To identify the most suitable CS acceleration factor, we performed 3D-FLAIR acquisitions, 1 without CS, and 4 with different CS factors $(1.2,1.3,1.4,1.5)$ on 5 healthy volunteers.

The FLAIR acquisitions were all identical except for the CS acceleration factor and the related scan time.

For each FLAIR acquisition, image quality was rated on a 3-point scale: 1, "poor" (image quality insufficient for diagnostic purposes); 2, "good" (minor artifacts not impairing diagnosis); and 3, "excellent" (no artifacts). We based the imagequality rating on 3 criteria: delineation of deep brain nuclei and the cortical gray-white matter junction, suppression of CSF, and blurring.

The purpose of this preliminary study in healthy volunteers was to identify the highest scan time reduction of 3D-FLAIR with CS compatible with an image quality score of at least 2 for all 3 criteria.
Table 2: Qualitative analysis ${ }^{\mathrm{a}}$

\begin{tabular}{lcccc}
\hline & JC & PV & IT & ON \\
\hline Reader 1 & & & & \\
$\quad$ Conventional FLAIR & 131 & 316 & 64 & 8 \\
$\quad$ CS FLAIR & 131 & 326 & 65 & 8 \\
$\quad$ Reader 2 & & & & \\
$\quad$ Conventional FLAIR & 140 & 373 & 76 & 10 \\
$\quad$ CS FLAIR & 129 & 377 & 75 & 10 \\
$\begin{array}{l}\text { Consensus reading } \\
\quad \text { Conventional FLAIR }\end{array}$ & 130 & 314 & 64 & 8 \\
$\quad$ CS FLAIR & 131 & 327 & 65 & 8 \\
\hline
\end{tabular}

Note:-JC indicates juxtacortical; PV, periventricular; IT, infratentorial; ON, optic nerve.

${ }^{a}$ No. of MS lesions per localization, on conventional FLAIR and CS FLAIR, according to both readers, and after consensus with a third reader

\section{Image Analysis}

Qualitative Analysis in Patients with MS. All FLAIR images were first anonymized and randomly interpreted. Then, all images were blindly and independently assessed by 2 neuroradiologists (S.T.-M. and A.S., with 9 and 4 years of experience, respectively) on the available workstation (Advantage Workstation 4.6; GE Healthcare). Each patient was analyzed only once per session, with either conventional FLAIR or CS FLAIR. The 2 independent blinded readings took place 2 weeks apart to avoid recall bias. Image quality was rated on the same 3-level scale used in the preliminary study. We also evaluated CSF suppression, delineation of deep brain nuclei, and the gray-white matter junction on the same scale.

For each FLAIR acquisition, the readers counted the number of visible MS lesions involving periventricular, intra-juxtacortical, infratentorial, and optic nerve regions (as used to assess spatial dissemination using the McDonald revised or Magnetic Resonance Imaging in Multiple Sclerosis criteria [MAGNIMS $]^{3}$ ). A lesion was defined as a focal T2-weighted hyperintensity surrounded by normal isointense white matter. Only lesions of $>3$ $\mathrm{mm}$ along the longest axis were considered. Confluent lesions without precise delineation were considered a single lesion.

Finally, discrepancies between the reviewers as to whether a lesion was present were resolved in consensus with a third rater with 15 years of experience (J.H.). Statistical analysis was based on postconsensus lesion counts (consensus reading in Table 2).

Quantitative Analysis in Patients with MS. Contrast-to-noise ratio (CNR) and SNR analyses were performed by 1 neuroradiologist (S.T.-M.). For each patient and each FLAIR acquisition, ROIs were drawn on axial reformatted views, within the following: 1) an MS lesion, 2) normal-appearing white matter, and 3) background noise. Care was taken to avoid lesion borders. SNR and $\mathrm{CNR}$ were calculated as follows: $\mathrm{SNR}=\mathrm{SI} / \mathrm{SD}_{\text {noise }}$ and $\mathrm{CNR}=$ $\left(\mathrm{SI}_{\text {lesion }}-\mathrm{SI}_{\mathrm{WM}}\right) / \mathrm{SD}_{\text {noise, }}$ where $\mathrm{SI}$ is the average signal intensity of the lesion or the WM and $\mathrm{SD}_{\text {noise }}$ is the $\mathrm{SD}$ of the noise.

An automatic postprocessing was additionally performed with Quantib Brain 1.2 software (Quantib, Rotterdam, Netherlands) based on de Boer et al. ${ }^{12}$ We analyzed 2 sets of series: 1) the BRAVO T1-weighted scan in combination with the conventional FLAIR scan, and 2) the BRAVO T1-weighted scan in combination with CS FLAIR scan. We used Quantib Brain to quantify the volume and the total number of brain MS lesions visible on both FLAIR sequences, based on the automatic segmentation of white 
matter hyperintensities. Using such postprocessing, we took all the MS lesions into account, including those involving the deep white matter.

\section{Statistical Analysis}

The Lin concordance correlation coefficient was used to assess interrater agreement and intrareader agreement for total MS lesion count and was interpreted as follows: poor agreement $(<0.90)$, moderate agreement $(0.90$ to $<0.95)$, substantial agreement $(0.95$ to $<0.99)$, and almost perfect agreement $(\geq 0.99) .{ }^{13}$ The Student $t$ test was used to compare image-quality scores between conventional and CS FLAIR. The Wilcoxon signed rank test was performed to test the null hypothesis that there was no significant difference in the total number of MS lesions between conventional and CS FLAIR, based on the radiologists' count. The level of significance was set to $P<.05$. The Lin concordance correlation coefficient was also used to evaluate the agreement between conventional and CS FLAIR in terms of the number of lesions detected in each region. Statistical analysis was based on postconsensus lesion counts.

The Student $t$ test was used to compare SNR and CNR values between conventional and CS FLAIR. The total volume of MS lesions between the 2 FLAIR scans, as measured by automatic white-matter hyperintensity segmentation, was compared using the Wilcoxon signed rank test.

\section{RESULTS}

Qualitative data are shown in Table 2.

\section{Preliminary Study in Healthy Volunteers}

Scan times of the different FLAIR acquisitions, without CS and with a CS factor of $1.2,1.3,1.4$, and 1.5 , were 5 minutes 15 seconds, 4 minutes 10 seconds, 3 minutes 50 seconds, 3 minutes 30 seconds, and 3 minutes 22 seconds, respectively. CS acceleration factor values of 1.4 and 1.5 were disqualified due to a poor delineation (rated 1) of the cortical gray matter junction. A CS factor of 1.3 provided the highest scan time reduction with good (mean score, 2.5) image quality. The CS factor 1.2 yielded only marginally improved image quality (mean score, 2.6) compared with the CS factor 1.3 and was 20 seconds shorter. As a result, the CS factor 1.3 was retained for the patient study.

\section{Qualitative Analysis in Patients with MS}

Interrater agreement as measured by the Lin concordance correlation coefficient was substantial (score of 0.95), and intrarater agreement was almost perfect (score, 0.99).

Image-quality scores did not differ between conventional and CS FLAIR $(P=.74)$. Mean image-quality scores were $2.78 \pm 0.42$ for conventional FLAIR, and $2.73 \pm 0.44$ for CS FLAIR. All FLAIR images were rated at least good. CSF suppression, delineation of the deep brain nuclei, and junction delineation were identical between every set of FLAIR images and rated as excellent. Of the 23 CS FLAIR acquisitions, 6 acquisitions were rated good instead of excellent due to a slight image blurring that did not impair diagnosis.

The total number of MS lesions detected by readers was similar between conventional and CS FLAIR (542 versus 557, $P=.094$ ).

The correlation coefficient between conventional and CS

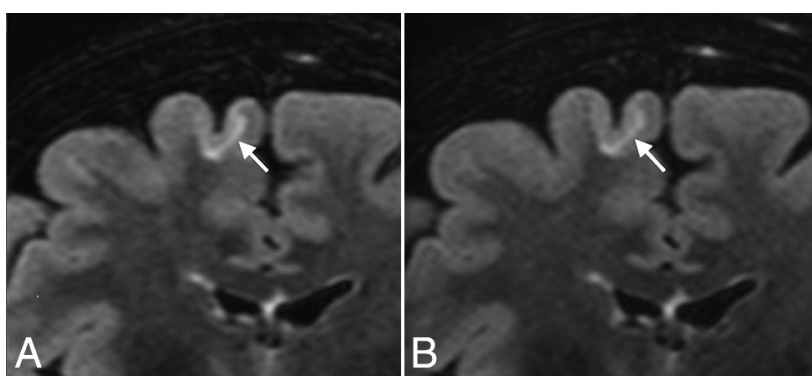

FIG 1. Coronal reformatted views of 3D-FLAIR with $(A)$ and without (B) CS, showing a juxtacortical lesion involving the right frontal superior gyrus (arrows) in a 50-year-old female patient with relapsingremitting MS. Note the similar delineation of the gray-white matter junction between conventional and CS FLAIR.

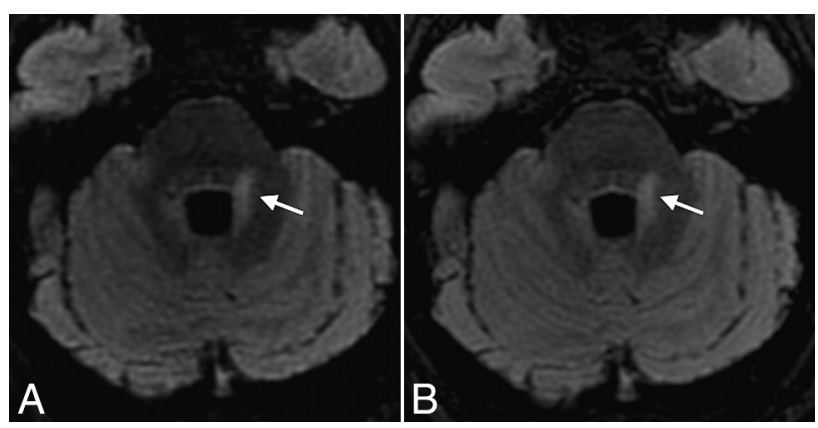

FIG 2. Axial reformatted views of 3D-FLAIR with $(A)$ and without $(B)$ $\mathrm{CS}$, showing a periventricular MS lesion also involving the left middle cerebellar peduncle (arrows) in a 31-year-old female patient with relapsing-remitting MS. Note the excellent and similar suppression of CSF obtained with both sequences.
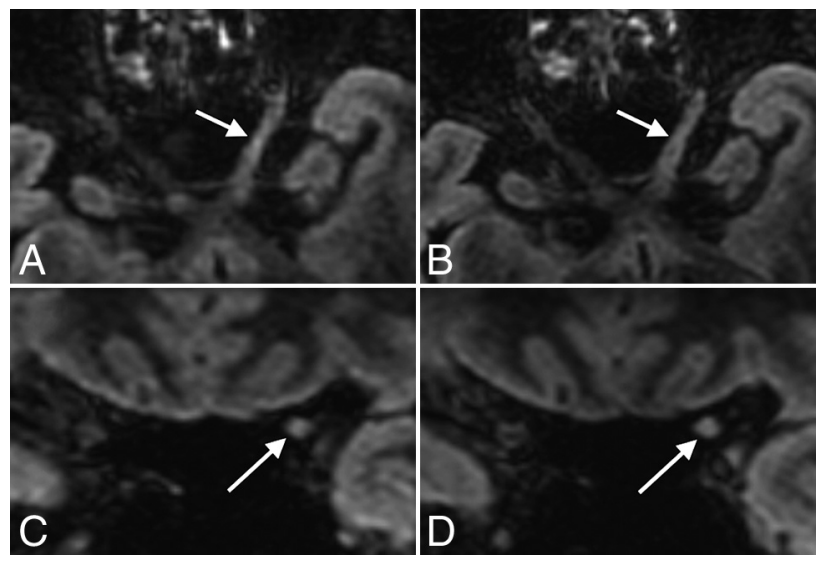

FIG 3. Axial and coronal reformatted views of $3 D$-FLAIR with ( $A$ and $C)$ and without $(B$ and $D) C S$, showing an MS lesion involving the cisternal and canalicular portions of the left optic nerve (arrows) in a 48-year-old female patient with relapsing-remitting MS.

FLAIR for the total MS lesion count after consensus was almost perfect (score, 0.99). Agreement between conventional and CS FLAIR was almost perfect for periventricular and infratentorial lesions (score, 0.99; Fig 2) and substantial for intra-juxtacortical and optic nerve lesions (score, 0.98; Figs 1 and 3 ).

\section{Quantitative Analysis in Patients with MS}

There was no statistical difference between the 2 FLAIR sequences in the SNR of normal-appearing white matter $(P=.57)$, the SNR 


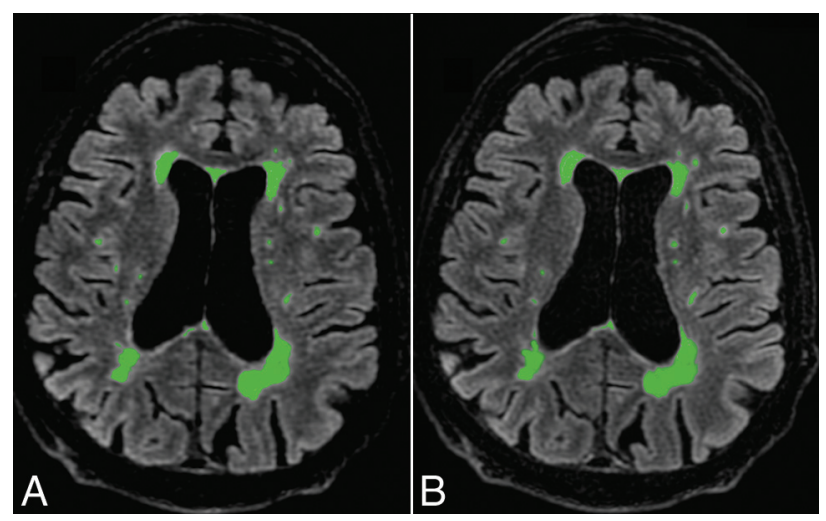

FIG 4. Automatic postprocessing performed with Quantib Brain 1.2 software allowing the assessment of MS lesion volumes using CS (A) and conventional FLAIR (B). With such a quantitative approach, FLAIR hyperintensities of the whole brain were taken into account. There was no significant difference between both FLAIR acquisitions in the total MS lesion volume $(P=.63)$ and number $(0.15)$.

of MS lesions $(P=.49)$, and the CNR of MS lesions to normalappearing white matter $(P=.52)$.

Postprocessing with Quantib Brain 1.2 software did not reveal any significant difference between conventional and CS FLAIR in total MS lesion volume $(P=.63)$ or in the number of MS lesions $(P=.15)$ automatically measured (Fig 4$)$.

\section{DISCUSSION}

Reducing the MR imaging scan time may improve patient comfort, cost-effectiveness, motion-related artifacts, and thus reproducibility. This improvement may be particularly useful for 3D FSE sequences, such as FLAIR, that usually require long acquisition times. In this study, we found that by adding CS to standard PI acceleration strategies, we can reduce the scan time of $3 \mathrm{D}$ FLAIR by $27 \%$ ( 1 minute 25 seconds) while preserving the accuracy of the detection of MS plaques. This result appears clinically relevant, considering the widespread use of 3D-FLAIR in MR imaging protocols dedicated to MS. ${ }^{4}$ Additionally, the agreement of results produced automatically by postprocessing further strengthens our conclusion, providing an unbiased analysis and taking into account all the visible MS lesions, including those involving the deep white matter.

To our knowledge, the combination of PI and CS to achieve FLAIR imaging has never been evaluated in patients with MS. The use of CS in neuroimaging has been evaluated in healthy volunteers with diffusion imaging ${ }^{14}$ and in patients with intracranial aneurysms using TOF-MRA. ${ }^{15}$ Our results are also fully concordant with recent studies evaluating CS in cardiac, abdominal, or knee imaging. ${ }^{16-19}$ Indeed, these studies have shown comparable image quality and lesion conspicuity between accelerated and nonaccelerated sequences. Admittedly, higher CS factors and scan time reductions can be achieved in $\mathrm{MR}$ sequences producing high contrast images with high data sparsity such as TOF-MRA, but such analysis is beyond the scope of the present study.

We observed image blurring in some of the CS FLAIR acquisitions. Such findings appear inherent to the CS technique that uses a variable random undersampling of the $k$-space, more pronounced at the periphery, thus leading to blurred images. While the quality of CSF suppression was identical in
CS FLAIR and conventional FLAIR, a slight difference in deep brain nuclei and gray-white matter junction delineation was observed due to minor motion-related blurring that did not affect the diagnostic performance. We used a simplified 3 -point scale to evaluate image quality. A more detailed scale may have exposed these minor differences, even though they have no impact on diagnostic performance. We performed accelerated FLAIR with a CS factor of 1.3 after testing a wide range of acceleration factors (detailed in the preliminary study) because this value appeared to us as a good compromise among blurring, reduced scan time, and accuracy.

In this study, we aimed at reducing the scan time of the 3D-FLAIR sequence with CS and evaluated its diagnostic performance. However, CS could also be used to further improve image quality in the same acquisition time of the conventional sequence by, for example, increasing the spatial resolution and/or contrast. Such an approach could be particularly useful to reduce partial volume effects and improve delineation of the cortical ribbon.

Of note, the diagnostic performance of both conventional and CS FLAIR appeared similar regardless of the topography of the inflammatory lesions, including those involving the optic nerves. Such findings may be particularly relevant in patients suspected of having MS and for whom spatial dissemination is customarily evaluated on the basis of FLAIR images. ${ }^{1}$

This study has some limitations. First, we did not include healthy controls since SNR values were also measured within the normal-appearing white matter of patients. Second, the number of MS lesions detected tended to differ between the 2 blinded readers (particularly for periventricular lesions). Such differences may be related to the small size of some lesions. However, both readers found no significant difference between conventional and CS FLAIR, whatever the lesion topography. We did not evaluate the impact of the acceleration factor value on diagnostic performance because we systematically applied a previously determined (by a preliminary study) CS factor of 1.3. Such a study design would have led to an important increase in protocol duration, which was not acceptable in a clinical setting. Further studies may investigate the optimal combination of acceleration factors of PI and CS according to the artifacts observed. Because we only included follow-up MRIs of patients with relapsing-remitting MS, we did not evaluate the performance of CS FLAIR in other types of MS, such as clinically isolated syndrome. However, it is reasonable to expect that the detectability of MS plaques using FLAIR may not differ according to MS types.

\section{CONCLUSIONS}

3D-FLAIR with a CS acceleration factor of 1.3 appeared to be the best compromise between scan time reduction and diagnostic performance in the detection of MS lesions.

Disclosures: Renske de Boer-UNRELATED: Employment: Quantib B.V., Comments: Quantib B.V. developed Quantib Brain; Royalties: GE Healthcare, Comments: Quantib B.V. receives royalties from GE Healthcare for Quantib Brain sales. * Jaap GelderblomUNRELATED: Employment: Quantib B.V., Comments: Quantib B.V. developed Quantib Brain; Royalties: GE Healthcare, Comments: Quantib B.V. receives royalties from GE Healthcare for Quantib Brain sales*. Rachid Mahdjoub_-UNRELATED: Employment: GE Healthcare, Comments: I am the neuroclinical leader; thus, I provide support and collaborate with research activities of our customers. *Money paid to the institution. 


\section{REFERENCES}

1. Polman CH, Reingold SC, Banwell B, et al. Diagnostic criteria for multiple sclerosis: 2010 revisions to the McDonald criteria. Ann Neurol 2011;69:292-302 CrossRef Medline

2. Aiken AH, Mukherjee P, Green AJ, et al. MR imaging of optic neuropathy with extended echo-train acquisition fluid-attenuated inversion recovery. AJNR Am J Neuroradiol 2011;32:301-05 CrossRef Medline

3. Filippi M, Rocca MA, Ciccarelli O, et al; MAGNIMS Study Group. MRI criteria for the diagnosis of multiple sclerosis: MAGNIMS consensus guidelines. Lancet Neurol 2016;15:292-303 CrossRef Medline

4. Moraal B, Roosendaal SD, Pouwels PJW, et al. Multi-contrast, isotropic, single-slab 3D MR imaging in multiple sclerosis. Eur Radiol 2008;18:2311-20 CrossRef Medline

5. Donoho DL. Compressed sensing. IEEE Transactions on Information Theory 2006;52:1289-306 CrossRef

6. Kayvanrad M, Lin A, Joshi R, et al. Diagnostic quality assessment of compressed sensing accelerated magnetic resonance neuroimaging. J Magn Reson Imaging 2016;44:433-44 CrossRef Medline

7. Geethanath S, Reddy R, Konar AS, et al. Compressed sensing MRI: a review. Crit Rev Biomed Eng 2013;41:183-204 CrossRef Medline

8. Lustig M, Donoho D, Pauly JM. Sparse MRI: the application of compressed sensing for rapid MR imaging. Magn Reson Med 2007;58: 1182-95 CrossRef Medline

9. Vasanawala SS, Alley MT, Hargreaves BA, et al. Improved pediatric MR imaging with compressed sensing. Radiology 2010;256:607-16 CrossRef Medline

10. Lustig M, Pauly JM. SPIRiT: iterative self-consistent parallel imaging reconstruction from arbitrary k-space. Magn Reson Med 2010; 64:457-71 CrossRef Medline
11. King $\mathrm{K}, \mathrm{Xu} \mathrm{D}$, Brau AC, et al. A new combination of compressed sensing and data driven parallel imaging. In: Proceedings of the Annual Meeting of the International Society for Magnetic Resonance in Medicine, Stockholm, Sweden. May 1-7, 2010:4881

12. de Boer R, Vrooman HA, van der Lijn F, et al. White matter lesion extension to automatic brain tissue segmentation on MRI. Neuroimage 2009;45:1151-61 CrossRef Medline

13. Lin LI. A concordance correlation coefficient to evaluate reproducibility. Biometrics 1989;45:255-68 CrossRef Medline

14. Menzel MI, Tan ET, Khare K, et al. Accelerated diffusion spectrum imaging in the human brain using compressed sensing. Magn Reson Med 2011;66:1226-33 CrossRef Medline

15. Fushimi Y, Fujimoto K, Okada T, et al. Compressed sensing 3-dimensional time-of-flight magnetic resonance angiography for cerebral aneurysms: optimization and evaluation. Invest Radiol 2016; 51:228-35 CrossRef Medline

16. Basha TA, Akçakaya M, Liew C, et al. Clinical performance of high-resolution late gadolinium enhancement imaging with compressed sensing. J Magn Reson Imaging 2017;46:1829-38 CrossRef Medline

17. Feng L, Benkert T, Block KT, et al. Compressed sensing for body MRI. J Magn Reson Imaging 2016;45:966-87 CrossRef Medline

18. Seo N, Park MS, Han K, et al. Feasibility of 3D navigator-triggered magnetic resonance cholangiopancreatography with combined parallel imaging and compressed sensing reconstruction at 3T. $J$ Magn Reson Imaging 2017;46:1289-97 CrossRef Medline

19. Kijowski R, Rosas H, Samsonov A, et al. Knee imaging: rapid threedimensional fast spin-echo using compressed sensing. J Magn Reson Imaging 2017;45:1712-22 CrossRef Medline 